\title{
GAIT VARIABILITY AND MOTOR CONTROL IN PATIENTS WITH KNEE OSTEOARTHRITIS AS MEASURED BY THE UNCONTROLLED MANIFOLD TECHNIQUE
}

\author{
Gwenllian Fflur Tawy ${ }^{\mathrm{a}}$ Philip Rowe ${ }^{\mathrm{a}}$ Leela Biant $^{\mathrm{b}}$ \\ a The Department of Biomedical Engineering, University of Strathclyde, \\ Wolfson Centre, 106 Rottenrow, Glasgow, G4 0NW, United Kingdom \\ ${ }^{\mathrm{b}}$ The Department of Trauma \& Orthopaedic Surgery, University of \\ Manchester, Manchester Royal Infirmary, Oxford Road, Manchester, M13 \\ 9WL, United Kingdom
}

\begin{abstract}
Knee osteoarthritis (OA) causes pain, reduced muscular strength and stiffness of the affected joint. In response, the motor control mechanism is altered, potentially compromising stability during acts of daily living. Reduced walking stability can be quantified in terms of gait variability. This study therefore aimed to identify and quantify the effects of knee arthritis on gait variability. Fifty adults (25 males/25 females) with end-stage OA of the knee sufficiently symptomatic to require joint replacement, walked on a self-paced treadmill for 2 minutes. A motion capture system was used to record 50 consecutive gait cycles from each patient. Kinematic variability of gait was analysed using the uncontrolled manifold technique (UCM). The position of the centre of mass (COM) was chosen as the task variable for the analysis. Results showed that our patient cohort were able to maintain a stable COM whilst walking, through adopting variable combinations of hip, knee and ankle kinematics. The greatest magnitudes of instability (based on the UCM ratios) occurred during initial contact and terminal stance. Active extension of the knee joint to approximately $5^{\circ}$ is required during these gait cycle events, meaning that these gait events are highly quadriceps dependent. This study identified and quantified components of the gait cycle
\end{abstract}


where patients with knee OA are most unstable. Employment of this technique could therefore allow specific personalised prescription for prehabilitation and rehabilitation.

Keywords: Uncontrolled manifold; Gait variability; Knee; Osteoarthritis 


\section{Introduction}

Approximately $23 \%$ of people over the age of 60 suffer from knee osteoarthritis (OA) [1]. Knee OA is a degenerative joint disease which causes pain, reduced muscular strength and limited function to the affected joint $[2,3]$. Consequently, patients suffering from late- to end-stage OA become limited in their mobility [1].

Koyama and colleagues stated recently that increased stiffness of the joint coupled with reduced muscular strength alters the motor control mechanism, ultimately compromising stability [4]. Stability can be quantified in terms of gait variability [5-7]. Although having a variable gait is natural, the extent of variability and the patterns observed in variability have been found to differ in those suffering from OA $[5,8,9]$. This could affect their ability to react to perturbations, potentially increasing the risk of falling and limiting the extent and speed of activity the patient feels safe to undertake $[7,10,11]$.

Given the complexity of gait, there are limited ways in which researchers and clinicians can objectively assess its overall variability from cycle-to-cycle $[7,12,13]$. Recent studies have used a method known as the uncontrolled manifold (UCM) hypothesis to investigate the relationship between motor control and variability in gait [14-16]. The populations in the studies by Papi et al. [14] and Black et al. [15] were stroke survivors and pre-adolescents with and without Down syndrome, respectively.

In these studies, the UCM method quantified the combinations of elemental variables (joint degrees of freedom) that successfully stabilised the centre of mass (COM): 'good variability', and those which compromised the stability of the COM: 'bad variability' [14, 15]. Here, stabilisation of the COM refers to the ability of the elemental variables to maintain a consistent mean COM 
position over numerous trials, despite showing inter-trial variability. Combinations of elemental variables that lead to a deviation of the COM away from its mean position compromise COM stability. Kinematic synergy was found to exist in each population in these studies, meaning that the 'good variability' (variance within the UCM) outweighed the 'bad variability' (variance orthogonal to the UCM) $[14,15]$. However, variability was increased compared to normal, implying that the central nervous system had employed a more variable gait in order to maintain a stable COM during walking. This strategy is believed to reduce COM instability, but to leave the subject more vulnerable to external or internal perturbations as some of the ability to variably respond to these perturbations has already been used $[17,18]$.

Improving our knowledge of the relationship between gait variability and COM stability in physically compromised populations may enable us to investigate the possibility of using the UCM method as a biomarker for gait stability and risk of falling.

The aim of this study was therefore to use the UCM method to quantitatively evaluate sagittal and frontal plane postural stability during normal walking in an osteoarthritic population.

We hypothesised that our population would display cycle-to-cycle variability when walking while maintaining kinematic synergy.

\section{Materials and Methods}

\subsection{Study Participants}

Fifty adults ( 25 males and 25 females) with end-stage osteoarthritis of the knee were recruited to this study (Age: $70 \pm 9$, Mass: $85.4 \pm 16.9 \mathrm{~kg}$, Height: $1.65 \pm 0.11 \mathrm{~m}$, BMI: $31.2 \pm 5.1 \mathrm{~kg} / \mathrm{m}^{2}$ ). All patients were scheduled to undergo total knee arthroplasty on the worst-affected knee. 15 patients 
had a valgus knee deformity (mean: $6.3 \pm 4.9^{\circ}$ ) and 35 had a varus knee deformity (mean: $\left.11.3 \pm 29.8^{\circ}\right)$. The contralateral knee was of similar OA severity in all patients. The study took place at the Royal Infirmary of Edinburgh, and was granted Ethics Approval as part of a wide-ranging study of total knee arthroplasty by the local research ethics committee (REC reference: 15/SS/0058). All patients gave verbal and written consent to participate in the study.

\subsection{Data Collection}

An eight-camera motion capture system was used to collect biomechanical data (Vicon, Oxford Metrics Ltd., UK). Three-dimensional co-ordinates of anatomical landmarks and lower limb kinematics were defined using the Strathclyde cluster biomechanical model. Joint co-ordinate systems and kinematics for this model were calculated as per International Society of Biomechanics recommendations [19-20]. Anatomical landmarks were calibrated with an instrumented pointer using the calibrated anatomical systems technique [21-22]. A diagram of the marker set used to create the model is shown in Fig. 1.

Patients were asked to walk on a 2-meter long treadmill for 2 minutes in shod conditions (Motekforce Link, Amsterdam, Netherlands). Walking speed was controlled by the patient through a 'self-paced' function; Treadmill belt speed increased as the patient walked towards the front of the treadmill (i.e. as the patient walked faster) and decreased as the patient moved towards the back of the treadmill (i.e. as the patient walked more slowly). This allowed the patient to adopt a walking speed that was natural to them. As a consequence, the variability of the gait calculated by our model is believed to be a good representation of true gait, which naturally varies in walking speed.

\subsection{Data Processing}


Anatomical landmark co-ordinates and kinematics of the hips, knees, and ankles were exported into text files post-assessment. All text files were imported into a custom-written MATLAB application which normalised the data to 101 points per cycle and applied the UCM to the data (ver. R2014b: MathWorks Inc., Massachusetts, US).

The chosen task variable was the COM trajectory, and the elemental variables were the foot-toground angles, and hip, knee and ankle kinematics in sagittal and frontal planes. The location of the COM was estimated as the geometric centre of the pelvis (using the co-ordinates from the left and right anterior and posterior superior iliac spines) [14]. Although the centre of the pelvis does not necessarily represent the position of the COM this method has been previously used, and was adopted in this study as a means of simplifying the complex model [14]. The elemental and task variables were linked through null spaces $\left(\mathrm{N}\left(\mathrm{J}_{\mathrm{S}}\right)\right.$ and $\left.\mathrm{N}\left(\mathrm{J}_{\mathrm{F}}\right)\right)$ of two Jacobian matrices $\left(\mathrm{J}\left(\theta_{\mathrm{S}}\right)\right.$ and $\mathrm{J}\left(\theta_{\mathrm{F}}\right)$ ); one for each plane (Equation 1 \& 2).

$$
\begin{gathered}
J\left(\theta_{S}\right)=\left[\begin{array}{l}
\frac{\delta x_{C O M}}{\delta \theta_{G}} \frac{\delta x_{C O M}}{\delta \theta_{A F}} \frac{\delta x_{C O M}}{\delta \theta_{K F}} \frac{\delta x_{C O M}}{\delta \theta_{H F}} \\
\frac{\delta y_{C O M}}{\delta \theta_{G}} \frac{\delta y_{C O M}}{\delta \theta_{A F}} \frac{\delta y_{C O M}}{\delta \theta_{K F}} \frac{\delta y_{C O M}}{\delta \theta_{H F}}
\end{array}\right] N\left(J_{S}\right)=\left[\begin{array}{l}
\varepsilon_{11}-\varepsilon_{12} \\
\varepsilon_{21}-\varepsilon_{22} \\
\varepsilon_{31}-\varepsilon_{32} \\
\varepsilon_{41}-\varepsilon_{42}
\end{array}\right] \\
J\left(\theta_{F}\right)=\left[\begin{array}{l}
\frac{\delta y_{C O M}}{\delta \theta_{G}} \frac{\delta y_{C O M}}{\delta \theta_{A A}} \frac{\delta y_{C O M}}{\delta \theta_{K A}} \frac{\delta y_{C O M}}{\delta \theta_{H A}} \\
\frac{\delta z_{C O M}}{\delta \theta_{G}} \frac{\delta z_{C O M}}{\delta \theta_{A A}} \frac{\delta z_{C O M}}{\delta \theta_{K A}} \frac{\delta z_{C O M}}{\delta \theta_{H A}}
\end{array}\right] N\left(J_{F}\right)=\left[\begin{array}{l}
\varepsilon_{11}-\varepsilon_{12} \\
\varepsilon_{21}-\varepsilon_{22} \\
\varepsilon_{31}-\varepsilon_{32} \\
\varepsilon_{41}-\varepsilon_{42}
\end{array}\right]
\end{gathered}
$$


Where $\mathrm{xCOM}, \mathrm{yCOM}$ and $\mathrm{zCOM}$ are the trajectories of the COM in $\mathrm{x}$-(anterior-posterior), $\mathrm{y}$ (vertical) and z-(medio-lateral) directions, and $\theta_{\mathrm{G}}, \theta_{\mathrm{AF}}, \theta_{\mathrm{KF}}$ and $\theta_{\mathrm{HF}}$ are the angles between the sole of the foot and ground, ankle flexion, knee flexion and hip flexion. $\theta_{\mathrm{G}}, \theta_{\mathrm{AA}}, \theta_{\mathrm{KA}}$ and $\theta_{\mathrm{HA}}$ are the equivalent in the frontal plane (ab-adduction). Intersegmental angles were calculated from the biomechanical model and the angle between the foot and the ground were calculated using Equation 3.

$$
\theta_{G}=\arcsin (\vec{a} \times \vec{b})
$$

Where $\vec{a}$ represents the vector of the sole of the foot (sagittal or frontal plane) and $\vec{b}$ represents the ground, as shown by Papi in 2015 [14].

Deviation matrices (created from the mean of each elemental variable per cycle subtracted from the respective elemental variable value at each percentage of the cycle) were then multiplied by the null spaces to give two vectors: one which was within the UCM (Equation 4), and one orthogonal to it (Equation 5).

$$
\begin{gathered}
\theta_{\|}=\sum_{i=1}^{n-d}\left(N(J)_{i}^{T} \cdot D M\right) N(J)_{i} \\
\theta_{\perp}=D M-\theta_{\|}
\end{gathered}
$$


The variances within ('good') and orthogonal to ('bad') the UCM were then calculated (Equations $6 \&$ 7). These represent the consistency of the elemental variables at each percentage of the gait cycle e.g. if the combinations of elemental variables led to a COM position which was consistent with the mean COM position (calculated over 50 gait cycles), the variance within ('good') the UCM would be greater than the variance orthogonal ('bad') to the UCM. This suggests that although the joint angles varied between cycles, the stable COM position was not affected. Alternatively, if the combinations of elemental variables led to a deviation of the COM away from its stable mean position, the variance orthogonal to the UCM ('bad') would be greater than the variance within ('good') the UCM.

$$
\begin{gathered}
\sigma_{\|}^{2}=(n-d)^{-1} \cdot(N)^{-1} \cdot \sum \theta_{\|}^{2} \\
\sigma_{\perp}^{2}=d^{-1} \cdot(N)^{-1} \cdot \sum \theta_{\perp}^{2}
\end{gathered}
$$

Where $\sigma_{\|}^{2}$ and $\sigma_{\perp}^{2}$ are the squared lengths of the vectors within and orthogonal to the UCM, respectively; $\mathrm{n}$ is the number of elemental variables $(\mathrm{n}=4) ; \mathrm{d}$ is the number of dimensions $(\mathrm{d}=2)$, and $\mathrm{N}$ is the number of gait cycles under analysis $(\mathrm{N}=50)$.

\subsection{Data Analysis}

Observation, graphical display, description and statistical analysis of the results were carried out in Minitab software (ver. 16: Minitab Inc., State College, PA, USA). The level of significance was set as $\alpha=0.05$. Non-parametric tests were carried out on groups of variances to determine whether kinematic synergy existed. Balanced ratios of variances within the UCM ('good variability') and 
orthogonal to the UCM ('bad variability) were also calculated (Equation 8). A ratio $>0$ suggests that the task in question (COM stabilisation) was successful as it indicates that the variance within the UCM ('good') outweighed the variance orthogonal to the UCM ('bad').As such, ratios above 0 imply that the variable joint kinematics adopted by the CNS over numerous consecutive gait cycles did not cause the position of the COM to deviate from its stable mean position.

$$
\text { RATIO }=\left(\frac{2 \theta_{\|}^{2}}{\theta_{\|}^{2}+\theta_{\perp}^{2}}\right)-1
$$

\section{Results}

Variances within the UCM were greater than the variances orthogonal to the UCM throughout the gait cycle in both planes and limbs (Fig. 2). This suggested that kinematic synergy existed in our patient cohort. In the sagittal plane, a prominent increase in variance was observed at approximately $80 \%$ of the gait cycle in the arthritic pre-operative limb. No such increase was observed in the non-operative limb. Frontal plane variance within the UCM in the arthritic preoperative limb was greater than that of the non-operative limb, peaking at $20 \%$ and $70 \%$ of the gait cycle. As in the sagittal plane, variance in the non-operative limb remained relatively constant throughout the gait cycle.

Statistical analyses were carried out to investigate whether variances within the UCM outweighed variances orthogonal to the UCM (Table 1). The results confirmed that kinematic synergy existed in our patient cohort to stabilise the COM when walking (Table 1). 
The success of COM stability was also confirmed by plotting the ratios of 'good' to 'bad' variances (Fig.3). The ratios were higher in the non-operative limb in both sagittal and frontal planes (Fig.3; Sagittal Plane: $\mathrm{p}=<0.0001$, Frontal Plane: $\mathrm{p}<0.0001)$.

Overall, these results suggested that certain stages of the gait cycle were more variable than others, but that this did not negatively impact the ability of the individuals to control the position of the COM whilst walking.

To further investigate this suggestion, results from a representative individual were analysed in greater detail. This individual was chosen randomly.

These results show that the variability of the angles in the sagittal plane (standard deviation bars) were greater in all joints of the operative limb than the non-operative limb (Fig.4). This was consistent with our previous findings (Fig.2). This relationship was not observed to the same extent in the frontal plane, except for at the ankle joint (Fig.4).

Kinematic variability in the frontal plane of our representative individual was relatively constant throughout the gait cycle (Fig.4). This was reflected in the frontal plane variances calculated (Fig.5).

Despite the variability observed, kinematic synergy was also shown to exist in the representative individual (Table 1). The COM was therefore effectively stabilised during the walking task in this patient. Most COM variability was observed in the antero-posterior direction. Least variability was observed in the medio-lateral direction. The degree of variability observed remained relatively consistent throughout the gait cycle. 


\section{Discussion}

The UCM hypothesis states that variability during repetitive movement tasks is necessary and beneficial. It enables us to act appropriately to perturbations or diseases which compromise elements of the kinematic chain of joints or which affect movement $[17,18]$. Patients with knee OA have generally been found to have alternative patterns of gait variability when compared to healthy adults, however the way in which the COM is stabilised to complete a walking task remained unclear $[5,7]$. This study applied the UCM method to gait data from 50 knee OA patients, with the aim to improve our understanding of gait variability and stability in this patient cohort.

Kinematic synergy was shown to exist in our patients during a walking task in both sagittal and frontal planes (Table 1). Our hypothesis can therefore be accepted. The variances reported in these individuals were greater on the pre-operative side (more symptomatic side) than the non-operative side, suggesting that the patients were adopting more variable gait kinematics on the pre-operative side, while maintaining COM stability when walking (Fig.2 \& Fig.5). A sudden peak in variance was observed in the sagittal plane during the swing phase of gait (Fig. 2 \& Fig.5). According to Remelius and colleagues [23], the body is most vulnerable to perturbations during this phase; therefore, increasing kinematic variability during swing phase may be a mechanism for maintaining a stable COM during a potentially unstable time. This mechanism was found to be successful in this patient cohort, as mean ratios were shown to increase with the variability and none tripped or fell while walking on the treadmill (Figs. 2-5).

Sagittal plane ratios were lowest at initial contact leading into loading response and terminal stance of the opposite limb (Fig.3). At initial contact, the knee is actively extended to $5^{\circ}$ by the quadriceps muscles. Body weight is then transferred onto the limb, where an extensor moment from the quadriceps muscles is again required in order to keep the knee stable and prevent collapse [24]. 
Terminal stance of the opposite limb also involves maximum extension of the knee joint [24]. These phases of the gait cycle are therefore quadriceps dependent. Given that individuals with knee OA have been shown to have reduced quadriceps strength it can be hypothesised that stability during gait could be improved in this patient population by focusing on quadriceps strengthening exercises [25].

Corroborating our theory is a study by Aljaker et al. [5] which showed that OA patients had increased soleus H-reflex amplitudes at the same phases of the gait cycle, suggesting increased use of the triceps surae muscle as a compensatory-mechanism due to poor quadriceps function. Although their study simultaneously showed that quadriceps activity was not significantly reduced in their OA population when compared to healthy age-matched controls, the level of knee OA of their patients was not severe enough to merit TKA [5].

In the frontal plane, variance within the UCM was highest during mid-stance and initial swing (Fig.3). During mid-stance, the COM shifts laterally, leaving the body weight vector to run medially through the knee joint [24]. Employing a more variable gait during this phase may therefore prevent the COM from moving too far laterally. It could also be a pain-avoidance mechanism, adopted by those with predominantly medial knee OA, as was the case in this sample, where $70 \%$ had a varus knee deformity. Although this study identified patients with valgus and varus knee deformities, the differences in gait variability between both groups were not investigated due to the small size of the valgus group. Future work will expand on this study to address this limitation.

Despite the fact that frontal plane ratios were relatively consistent throughout the gait cycle (Fig.3), slightly higher ratios were detected during mid-stance and initial swing phases, suggesting that a higher degree of variance was beneficial in stabilising the COM mediolaterally. 
In general, more kinematic variability was exhibited in the sagittal plane than frontal the plane, suggesting that a higher degree of variability was employed during gait to maintain a stable COM in this plane in our population. Some of this kinematic variability may be explained by the freedom of the patients to alter their walking speed during trials. However, given that walking speed naturally fluctuates, we are confident that the data presented here is a better representation of gait variability than if we had imposed a fixed walking speed on the patients.

Given the novelty of this study, our data cannot be compared directly to previously published literature. However, our study did show similarities to others that applied the UCM method to gait data $[14,15]$. As in our study, Papi et al. and Black et al. showed that kinematic synergy existed in stroke survivors and pre-adolescents with Down syndrome, suggesting that people with pathological gait were able to maintain a stable COM through employing variable gait kinematics $[14,15]$. Stroke survivors were on average shown to have higher sagittal plane ratios during stance than knee OA patients, but OA patients had greater stability at foot strike [14]. Pre-adolescents with Down syndrome had similar ratios during foot strike to knee OA patients [15]. When compared to healthy older adults, knee OA patients were found to have lower sagittal plane ratios during the stance phase of gait [14].

When devising our experimental protocol, we were aware of the differences that arise in some biomechanical parameters between over-ground and treadmill gait. We did not believe it appropriate to record over-ground walking in this study as tens to hundreds of consecutive cycles must be recorded to report gait variability. Hence, a large amount of space and additional motion capture equipment would have been required to record 50 consecutive cycles. To optimise our treadmill protocol, a long $(2 \mathrm{~m})$ treadmill was used. This was deemed suitable as short treadmill lengths have been ascribed to some of the differences in biomechanical parameters of treadmill 
gait (Alton et al., 1998; Sloot et al., 2014; Strathy et al., 1983). We are therefore confident that the methodology used in this study represented gait variability in elderly OA individuals to the best ability.

One way in which our methodology could be improved in future is by calculating each body segment COM with respect to its mass then summating the results for a better representation of the COM.

\section{Conclusion}

A variable gait is employed to stabilise the centre of mass in patients with OA of the knee. Kinematic synergy was confirmed in this population. Weakness of the quadriceps is thought to decrease sagittal plane stability in this patient cohort. This technique may allow specific personalised prescription for prehabilitation and rehabilitation of knee OA patients.

\section{Acknowledgements}

This study was supported by the University of Strathclyde and Medacta International SA as a PhD studentship. Neither had direct involvement with this study or the writing of this manuscript.

\section{Conflict of Interest}

We have no conflict of interest to declare.

\section{References}


1. Heidari B. Knee osteoarthritis prevalence, risk factors, pathogenesis and features: Part I. Caspian J Intern Med 2011; 2(2): 205-212

2. Shenoy R, Pastides PS, Nathwani, D. (iii) Biomechanics of the knee and TKR. Orthop Trauma $2013 ; 27(6): 364-371$

3. Urwin M, Symmons D, Allison T, Brammah T, Busby H, Roxby M, Simmons A, Williams G. Estimating the burden of musculoskeletal disorders in the community: the comparative prevalence of symptoms at different anatomical sites, and the relation to social deprivation. Ann Rheum Dis 1998; 57(11): 649-655

4. Koyama Y, Tateuchi H, Nishimura R, Ji X, Umegaki H, Kobayahsi M, Ichihashi N. Relationships between performance and kinematic/kinetic variables of stair descent in patients with medial knee osteoarthritis: An evaluation of dynamic stability using an extrapolated center of mass. Clin Biomech 2015; 30(10), 1066-1070

5. Alkjaer T, Raffalt PC, Dalsgaard H, Simonsen EB, Petersen NC, Biddal H, Henriksen M. Gait variability and motor control in people with knee osteoarthritis. Gait Posture 2015; 42(4):479484

6. Herman T, Giladi N, Gurevich T, Hausdorff J. Gait instability and fractal dynamics of older adults with a "cautious" gait: why do certain older adults walk fearfully? Gait Posture 2005; 21(2): $178-185$

7. Mahmoudian A, Bruijn S, Yakhdani HRF, Meijer OG, Verschueren SMP, van Dieen JH. Phasedependent changes in local dynamic stability during walking in elderly with and without knee osteoarthritis. J Biomech 2015; 49(1): 80-86

8. Bernstein NA. The co-ordination and regulation of movements. Pergamon Press: Oxford; 1967.

9. Kiss RM. Effect of severity of knee osteoarthritis on the variability of gait parameters. J Electromyogr Kinesiol 2011; 21: 695-703 
10. Lin X, Meijer OG, Lin J, Wu W, Lin X, Liang B, van Dieën JH, Bruijn SM. Frontal plane kinematics in walking with moderate hip osteoarthritis: Stability and fall risk. Clin Biomech $2015 ; 30(8), 874-880$

11. Maki BE. Gait changes in older adults: predictors of falls or indicators of fear. J Am Geriat Soc 1997; 45(3), 313-320

12. Hausdorff JM, Rios DA, Edelberg HK. Gait variability and fall risk in community-living older adults: a 1-year prospective study. Arch Phys Med Rehab 2001; 82(8):1050-1056

13. Riva F, Bisi M, Stagni R. Gait variability and stability measures: minimum number of strides and within-session reliability. Comp Biol Med 2011; 50: 9-13

14. Papi E, Rowe PJ, Pomeroy VM. Analysis of gait within the uncontrolled manifold hypothesis: Stabilisation of the centre of mass during gait. J Biomech 2015; 48(2), 324-331

15. Black DP, Smith BA, Wu J, Ulrich BD. Uncontrolled manifold analysis of segmental angle variability during walking: Preadolescents with and without Down syndrome. Exp Brain Res $2007 ; 183,511-521$

16. Qu X. Uncontrolled manifold analysis of gait variability: effects of load carriage and fatigue. Gait Posture 2012; 36(2), 325-329.

17. Latash ML, Scholz JP, Schöner G. Towards a new theory of motor synergies. Motor Control 2007; 11(3), 276-308

18. Cashaback JGA, McGregor HR, Gribble PL. The human motor system alters its reaching movement plan for task irrelevant, positional forces. J Neurophysiol 2015; 113, 2137-2149

19. Grood ES, Suntay W. A Joint Coordinate System for the Clinical Description of ThreeDimensional Motions. Application to the Knee. Transactions of the American Society of Mechanical Engineers 1983; 105, 136-144

20. Wu G, Cavanagh PR. ISB Recommendations in the Reporting for Standardization of Kinematic Data. J Biomech 1995; 28(10), 1257-1261. 
21. Cappozzo A, Catani F, Della Croce U, Leardini A. Position and orientation in space of bones during movement: anatomical frame definition and determination. Clin Biomech 1995; 10(4), 171-178.

22. Tawy GF, Rowe PJ. Is the instrumented pointer method of calibrating anatomical landmarks in 3D motion analysis reliable? J Biomech 2017; 53, 205-209

23. Remelius JG, Hamill J, van Emmerik REA. Prospective dynamic balance control during the swing phase of walking: stability boundaries and time-to-contact analysis. Hum Mov Sci 2014; $36,227-245$

24. Perry J \& Burnfield JM. Gait Analysis. Normal and Pathological Function. $2^{\text {nd }}$ ed. NJ USA: SLACK Incorporated; 2010.

25. Murray AM, Thomas AC, Armstrong CW, Piterosimone BG, Tevald MA. The associations between quadriceps muscle strength, power, and knee joint mechanics in knee osteoarthritis: A cross-sectional study. Clin Biomech 2015; 30(10), 1140-1145 


\section{Figure Captions}

Figure 1: Diagram of the marker set used to create the Strathclyde Cluster Model. Twenty anatomical landmarks are defined to calculate hip, knee and ankle kinematics.

Figure 2: Mean variance within (UCM) and orthogonal (ORT) to the linearized UCM in sagittal and frontal planes $(\mathrm{n}=50)$.

Figure 3: Mean ratios ( \pm standard deviation) in sagittal and frontal planes across 50 adults with knee OA.

Figure 4: Mean ( \pm standard deviation) hip, knee and ankle angles in sagittal and frontal planes in one representative individual over 50 gait cycles.

Figure 5: Variances within (UCM) and orthogonal (ORT) to the linearized UCM in sagittal and frontal planes from one representative individual. 


\begin{tabular}{l|l|} 
The Strathclyde Cluster Model: \\
$1 \times$ Pelvis cluster \\
$2 \times$ Thigh clusters \\
$2 \times$ Shank clusters \\
$2 \times$ Foot clusters \\
\\
$2 \times$-Anatomical Landmark \\
$2 \times$ Posterior Superior Iliac Spine \\
$2 \times$ Greater Trochanter \\
$2 \times$ Lateral Epicondyle \\
$2 \times$ Medial Epicondyle \\
$2 \times$ Lateral Malleolus \\
$2 \times$ Medial Malleolus \\
$2 \times$ Heel \\
$2 \times 1^{\text {st }}$ Metatarsal \\
$2 \times 5^{\text {th }}$ Metatarsal \\
\hline
\end{tabular}



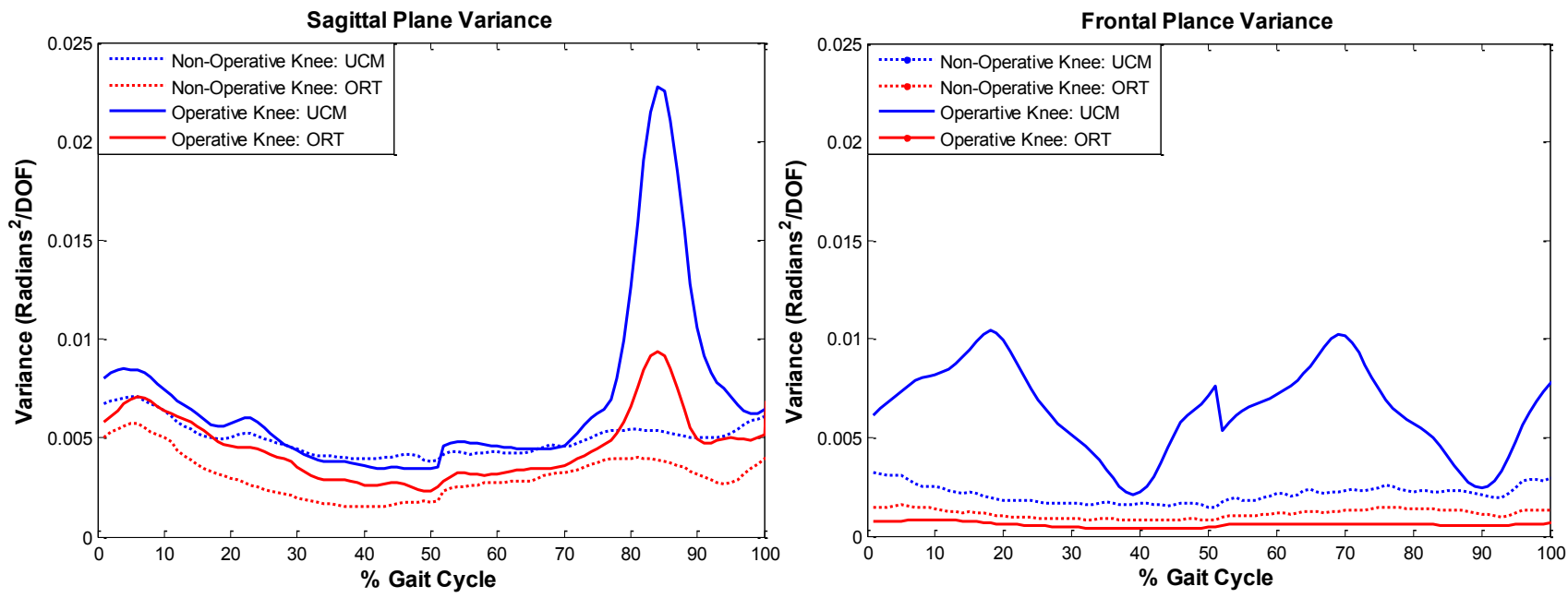

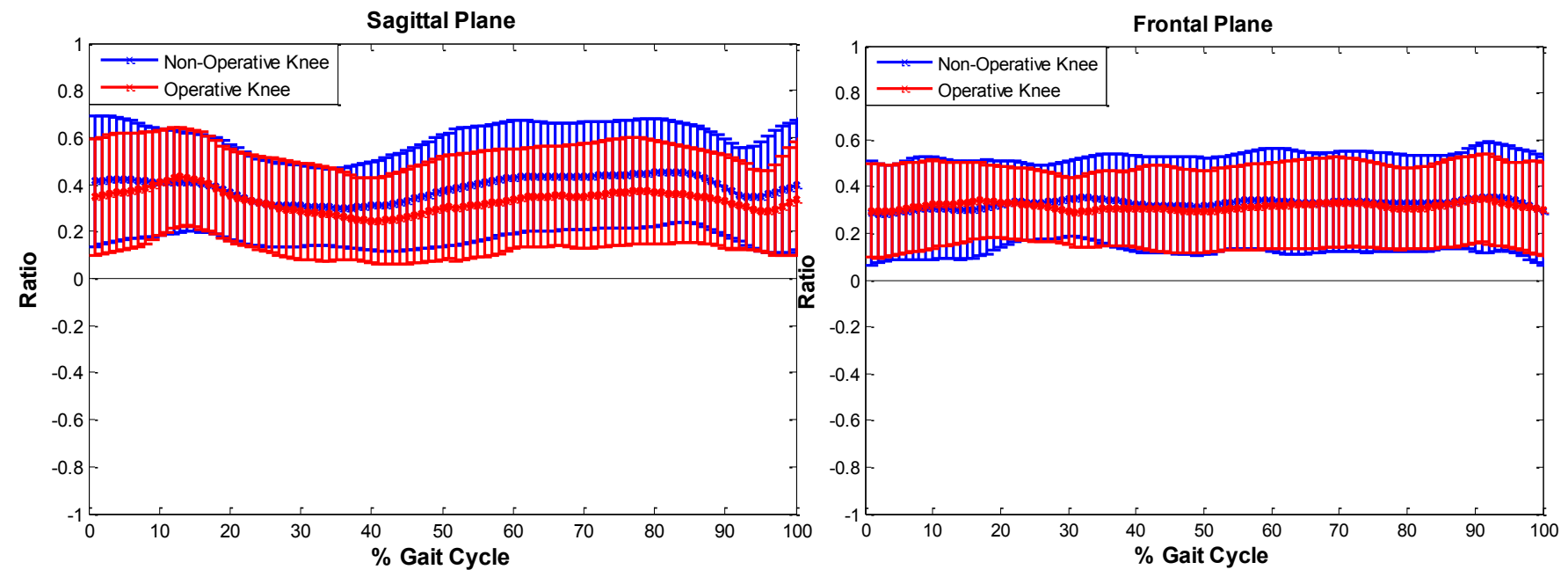

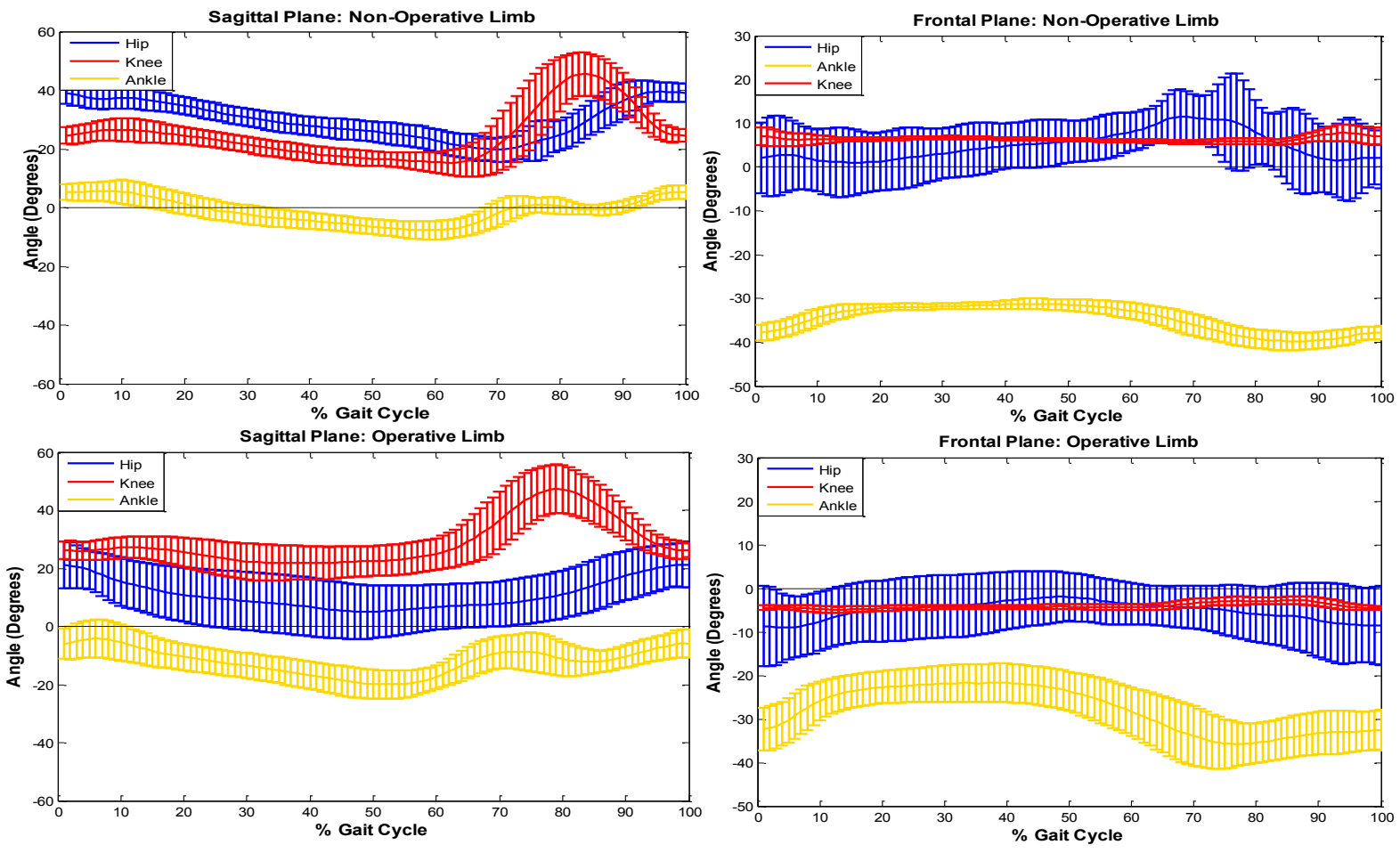

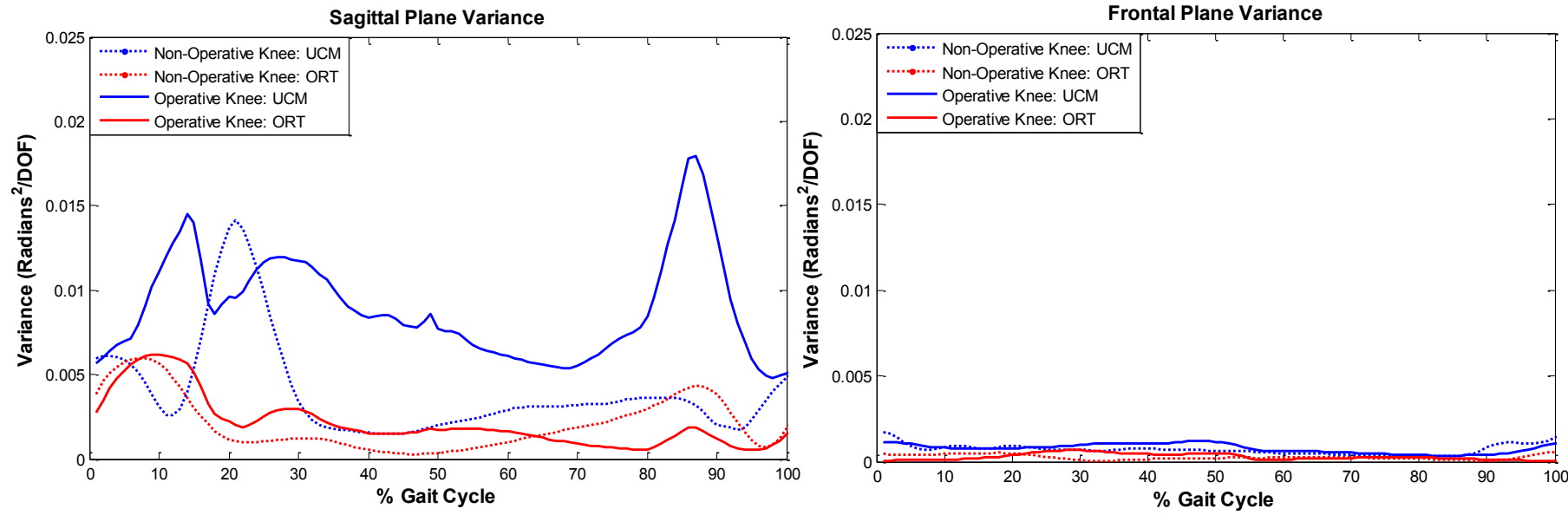
Table 1: Statistical comparison of variances recorded during walking.

\begin{tabular}{cccc}
\hline \multirow{2}{*}{ Side } & Analysis & \multicolumn{2}{c}{ p-value } \\
\cline { 3 - 4 } & & All Patients & Representative \\
& & & Individual \\
\hline \multirow{2}{*}{ Non-affected Limb } & Sagittal Plane UCM Variance vs. ORT Variance & 0.001 & 0.002 \\
\cline { 2 - 4 } & Frontal Plane UCM Variance vs. ORT Variance & $<0.001$ \\
\hline \multirow{2}{*}{ Affected Limb } & Sagittal Plane UCM Variance vs. ORT Variance & 0.001 \\
\cline { 2 - 4 } & Frontal Plane UCM Variance vs. ORT Variance & $<0.001$ & 0.001 \\
& & & 0.006 \\
\hline
\end{tabular}

